Session 1663

\title{
Addressing Multicultural Issues in Manufacturing Curricula
}

\author{
Murali Krishnamurthi \\ Northern Illinois University
}

\section{Introduction}

According to the National Science Foundation's report [4] on "Women, Minorities, and Persons with Disabilities in Science and Engineering," the enrollment figures on non-white students in science and engineering majors is around $10 \%$ and this figure is considerably small compared to the enrollment figures for the same category in non-science and engineering majors. If this enrollment trend continues the composition of future work force in technology-oriented fields will not truly reflect the multicultural make-up of our society. Considering the projected statistics on the composition of the future work force, it is imperative that educational institutions improve their science and engineering curricula to address gender equity and multicultural issues and help students experience, understand, and appreciate the benefits of the same before joining the work force [1].

The following were some of the concerns expressed to the author at the 1996 ASEE conference by some engineering faculty about addressing gender equity and multicultural issues in their curricula: (1) gender equity and multicultural issues appear to be more suited for addressing in the liberal arts curricula than the science and engineering curricula, (2) science and engineering courses do not seem to lend themselves to addressing these issues since the course content do not deal with these issues, (3) by addressing these issues, we may not be able to cover the required course material, and (4) we have to prepare students for the industry and the best way to do this is to let them deal with these things by themselves. These concerns may or may not be shared by all faculty in science and engineering curricula, but they certainly demonstrate the need to address them and clarify some of the misconceptions faculty may have about these issues.

\section{Steps For Multiculturally Transforming the Manufacturing Curricula}

Any type of transformation activity is difficult, especially the ones that deal with educating ourselves, changing our beliefs and attitudes, and changing the way we do things. Further, we are often told "what" to do, but not "how" to do it. Faculty, especially engineering faculty due to their scientific background and training, need a structured step-by-step methodology to follow on "how" to transform to their curricula, along with being told "what" to do. After participating in a multicultural curriculum transformation institute at Northern Illinois University during summer 1996, I began exploring how to transform my courses in manufacturing and related topics to address gender equity and multicultural issues and realized that I also needed a structured methodology to follow and that other faculty could also benefit from one. The following is a step-by-step methodology I have developed for multiculturally transforming any curricula. Step 1. Educate Yourself - Before attempting to analyze and transform our curricula, we should educate ourselves first on gender equity and multicultural issues. Educating ourselves requires keeping an open mind and giving ourselves the time to learn and examine the relevant issues. We 
can educate ourselves by attending institutes and seminars offered nowadays at most universities and at conferences such as ASEE, acquiring and reading the literature from journals such as ASEE Prism and the Journal of Engineering Education which periodically publish articles on these issues, discussing with our colleagues knowledgeable in these issues, interacting with a diverse group of students, and exploring the statistics on the demographics of the workforce.

Step 2: Analyze - Once we recognize that our curricula can be transformed, the next step is to analyze what exactly needs to be transformed. This requires analyzing the various aspects of our curricula, such as our teaching styles, students' learning styles, classroom interaction, course material selection, instructional design, course activities, student feedback, etc. These should be analyzed with the gender equity and multicultural perspectives in mind and the results of this analysis will help us in identifying opportunities for improvement. For example, teaching styles and student learning styles can be analyzed using inventories such as the Kolb inventory, and course material selection and instructional could be analyzed with the help of external reviewers. Classroom interaction and student feedback could be analyzed using assessment instruments.

Step 3: Identify Resources - After identifying the opportunities for improvement in our curricula, often we may need the necessary resources to transform our curricula. This may include curriculum development materials, videos and films, external speakers, books and journal articles, internet resources, etc. After I realized the opportunities for transforming my courses, I was able to find numerous resources on the internet and have compiled and made them available on my web page at "http://www.ceet.niu.edu/faculty/murali/useful.htm". With very little effort, I was able to search the internet and find case studies, examples, and news articles that I could use in my courses. I also maintain a list of women and minority speakers who I can call for giving presentations in my classes. There are also catalogs of videos available on multicultural issues from commercial educational video producers and trainers.

Step 4: Explore Course Content - One of the common misconceptions among science and engineering faculty is that the content of our courses is not suited for addressing gender equity and multicultural issues. This may be true for some engineering courses, but not necessarily for all courses. Unless we explore the content of our courses thoroughly we cannot identify the opportunities for addressing gender equity and multicultural issues. Course content can be explored from several angles such as the source and origin of inventions, design considerations based on gender/age/culture, application issues, facts and statistics used in the courses, etc. Once I began exploring my courses in engineering, I was quite surprised to find out how easily I could address gender equity and multicultural issues through course content.

For example, in my undergraduate operations research course I have used numerous historical facts to illustrate where many of the mathematical discoveries covered in the course came from and this helps students to understand that many cultures contributed to the development of ideas in the field. In my simulation course I have assigned a laboratory exercise for my students to simulate the use of restroom facilities by male and female workers in a manufacturing facility and comment on the results. This assignment was based on an actual news story related to the problems caused by insufficient number of restroom facilities in a manufacturing plant in Orange County, California. Lack of adequate restroom facilities in public building, theaters, sports arena, 
and manufacturing facilities is a common problem experienced by women and this assignment helped student to understand the need for addressing gender issues in facility design and become more aware of gender equity in facility design.

Manufacturing topics such as product design, marketing, and production management are very conducive for addressing gender equity and multicultural issues in the course content. When designing consumer products ranging from simple household appliances to automobiles, it is important to consider cultural issues, gender differences, and age of users. Cultural issues can significantly impact the sale of a product if the product will be marketed in other countries. For example, a simple product such as a can opener sold in U.S. may not find a large market in countries such as China or India since canned goods are not popular in these countries. Gender differences can also impact product usage since there are reach and height differences between genders, and age can be a factor if the product is designed to be used by a particular age group. Cultural issues can also impact how a product manufactured in U.S. can be marketed in other countries. Relocation of manufacturing industries abroad and the impact of their pollution on the environment are also suitable topics for addressing social issues through manufacturing courses.

Production management is a topic that has ample opportunities for addressing gender equity and multicultural issues. Production managers often have to consider age and gender issues when designing particular tasks on the manufacturing shop floor. Tasks that involve heavy lifting, repetitive motions, or working in a high temperature area or toxic environment may require addressing age and gender issues. Scheduling of production shifts through day and night and its impact on people is another topic that can be used to highlight social and cultural issues. These issues, however simple they may appear, are very relevant in a manufacturing course and it is easy to explain the impact of these issues from course content.

Human factors or ergonomics in manufacturing is another area that is ripe with opportunities for addressing gender and multicultural issues through topics such as work place design, job design, worker performance standards, job safety, etc., that impact humans both physiologically and psychologically. Work place design, for example, requires addressing differences in body dimensions and reach between genders, and job design requires addressing differences in average lifting capabilities between genders. Culture, gender, and age play a significant part in human factors, and therefore, this area very much suited for addressing gender and multicultural issues from the content perspective.

Step 5: Explore Course Materials - The materials we use in our courses can have a tremendous impact on what we convey to our students and the emphasis we place on gender equity and multicultural issues. Course materials can be selected/designed to eliminate biases of various kinds, highlight power and privilege issues, introduce balanced treatment of diversity, identify stereotyping of various kinds, point out societal attitudes and perceptions, and promote student's thinking on such critical issues. After I explored the text books in my field, I was able to find numerous examples of gender bias, cultural stereotyping, references to underage drinking, etc. If we do not carefully scrutinize our course materials before prescribing them for student use, we could be easily sending the wrong message to our students. The textbooks also contained little or no references to successful women or minority scientists and engineers, and to counter this bias, I 
selected from various sources [3, 7] biographies of successful women or minority scientists and engineers and included them in the handouts I prepared for my students.

Step 6: Design Course Activities - If course content is not suitable for addressing gender equity and multicultural issues, course activities can be used as a mechanism for addressing these issues. Course activities can include carefully designed in-class and outside class activities, homework assignments, tests, team projects, laboratory exercises, and presentations by women and minority speakers. For in-class and outside class activities there are a variety of cooperative learning techniques available which can promote respect among students for other cultures, opposite gender, and individual differences in learning.

In engineering classes women and minority students generally do not speak up or participate and class discussions are most often dominated by male students. The cooperative learning techniques give everyone an equal opportunity to participate, and be heard and respected. I have used a variety of cooperative learning techniques [6], such as the Think-Share Pair (TSP) and Think Aloud Problem Solving (TAPS) exercises for my in-class activities. The TSP exercise requires pairs of students to first think about a problem individually and then solve the problem as a pair using their combined solutions. The TAPS exercise requires one student in a pair to act as the listener and the other student as the problem solver. The problem solver is required to explain orally how he/she is solving the problem while being prompted by the listener to keep verbalizing his/her approach. The purpose of these exercises is that they give individual students an opportunity to speak up and be heard, and this promotes respect and equality among students.

I have learned from my past mistakes that care should also be exercised in designing homework assignments and tests so that gender bias and cultural stereotyping could be avoided. For one of my homework assignments in my information systems course I had asked students to draw a data flow diagram of the process of making Tacos. I thought students could benefit from doing this assignment for a simple process and that they could apply the same procedure for modeling a complicated engineering problem. Soon I realized that many of the international students in my class were not only unfamiliar with making tacos but also had not even tasted a taco!

In one of the midterm examination for my information systems course I had asked students to design a data model of an information system for maintaining information on "pot holes." During the exam I realized, from the look on the faces of international students, that they had not heard of the term "pot holes" in reference to holes on the road, and I had to explain it to them during the exam, wasting their valuable time. Since then I have made sure not to assume that every one of my students is familiar with popular sports or colloquial terminology, and I make a conscientious effort to design homework assignments and tests that are fair to everyone. For team projects in my courses, I usually decide the makeup of each team so that the composition of each team is balanced in terms of gender and cultural diversity. At the completion of team projects, I have assessed students' experiences in interacting with their project teammates and students' responses have only been positive about their experiences. Another course activity that is very relevant to this discussion is the use of women or minority speakers in the classroom. Engineering majors usually do not have the opportunity to see and listen to successful women or minority scientists and engineers and inviting them to give presentations in a science or 
engineering class can only help to promote a positive image of women and minorities in science and engineering. The mentioned activities can be introduced in all science and engineering courses, regardless of their content, to promote gender equity and diversity.

Step 7: Design Classroom Interaction - Classroom interactions between faculty and students, and among students can be tailored to promote gender equity and multicultural sensitivity. Faculty should ensure that classroom interactions are balanced to accommodate women and minority students and their differences in learning styles. It is important not to make examples of any group or put them on the spot to shoulder the responsibility of speaking for or representing their group. When using proper nouns in case studies or examples, care should be exercised to use names that represent various groups and gender. I make a conscious attempt in my classes to solicit the opinions of women and minority students to show that I value their opinions.

Step 8: Analyze Subtle Signals - Our sensitivity towards gender equity and diversity can be conveyed through subtle signals we send, such as our office decorations, cartoons and news articles that we post on bulletin boards and on our office doors, books we keep on our shelves, our memberships in social clubs, professional societies and service organizations, our informal conversations outside classroom settings, etc. I have decorated my office with very inexpensive artwork and crafts from various countries to show my openness towards other cultures and my interest in learning more about them.

Step 9: Examine Course Administration - One of the places we can make explicit statements about our views and policies on gender equity and multicultural issues is in the course syllabus. If appropriate, policies can be established to accommodate the observance of religious and cultural events.

Step 10: Evaluate - One of the key components of curriculum design is assessment, and along with including gender equity and multicultural activities in our curricula, we should also assess their effectiveness. This requires defining the objectives of each activity clearly, informing the administrators and curricular bodies about the activities, identifying criteria for measurement, developing mechanisms for measurement, conducting formal assessments, analyzing the assessment data, making valid conclusions about the effectiveness of the activities, and feeding back the results appropriately into our curricula.

Step 11: Plan for Continuous Improvement - Curriculum transformation is an ongoing process and we should plan for continuously improving our courses. The results we obtain from our assessment activities generally help us to explore the opportunities for improving the courses. It is helpful to obtain outside critique of all aspects of our courses.

Step 12: Educate Others - Once we have transformed our curricula and have achieved some success, it is essential that we take the message to others. It is our responsibility to educate others and help them recognize the value in addressing gender equity and multicultural issues. We can do this by presenting our success stories in conferences and professional meetings, publishing journal articles, and pursuing research in this area. These activities also have definite rewards for those of us in academia. 


\section{Conclusions}

The twelve steps mentioned in this paper are applicable not only to the manufacturing curricula, but also to any curricula. The key step is the first one which deals with our willingness to educate ourselves and to explore the possibilities. My exploration of the courses I teach in engineering has clearly shown that engineering courses are indeed suitable for addressing gender equity and multicultural issues, both from the content and course activities angles. The surveys I conducted in my classes indicate that my students appreciate the attention I give to gender equity and multicultural issues and my interest in raising their awareness of these issues.

I certainly recommend other faculty in engineering to explore their curricula and transform them to address gender equity and multicultural issues. There are also numerous resources currently available, especially on the internet, to aid faculty in this effort. Curricular transformations that support gender equity and diversity will certainly strengthen the curricula and attract more women and minorities into engineering fields, and this will only benefit the society as a whole.

\section{References}

[1] DiTomaso, N., and Farris, G. F., "Diversity in the High-Tech Workplace," IEEE Spectrum, pp. 20-32, June 1992.

[2] Johnston, W. B., and Packer, A. H., "Workforce 2000: Work and Workers for the Twenty-First Century," Report, Hudson Institute, Box 26-919, Indianapolis, Indiana, 1987.

[3] Mozans, H. J., “Woman in Science,” University of Notre Dame Press, London, 1991.

[4] National Science Foundation. "Women, Minorities, and Persons with Disabilities in Science and Engineering," Report, Washington, D.C., 1994.

[5] Phillips, D. T., et al. “Operations Research: Principles and Practice,” John Wiley \& Sons, New York, 1976.

[6] Whimbey, A., and Lochhead, J. “ Problem Solving and Comprehension,” Fifth Edition, Lawrence Erlbaum Associates, Publishers, New Jersey, 1991.

[7] Yost, E., “American Women of Science,” Frederick A. Stokes Publishers, Philadelphia, 1955.

\section{ACKNOWLEDGMENTS}

This effort was partially funded by a Curriculum and Course Development Grant, DUE/CCD 94-55748, awarded by the National Science Foundation to Northern Illinois University during 1995-1996. The author would like to express his gratitude to Dr. Kay Forest of the Department of Sociology at Northern Illinois University for her valuable suggestions for improving this paper and to the Multicultural Task Force at Northern Illinois University for the opportunity to attend the Multicultural Curriculum Transformation Institute held during summer 1996.

\section{MURALI KRISHNAMURTHI}

Murali Krishnamurthi is an Assistant Professor in the Department of Industrial Engineering at Northern Illinois University. He received his Ph.D. in Industrial Engineering from Texas A\&M University. His teaching and research interests are in Simulation, Manufacturing, Operations Research, Information Systems, and educational methods. He serves as a member of the Provost's Multicultural Task Force at Northern Illinois University. 